\title{
Progression of COVID- I 9 Pandemic in India: A Linear Functional Concurrent Regression Analysis Approach
}

\author{
Aalok Ranjan Chaurasia', Brijesh P Singh ${ }^{2}$, Ravendra Singh ${ }^{3}$ \\ ${ }^{1}$ President, MLC Foundation, Bhopal, Madhya Pradesh, India. \\ ${ }^{2}$ Professor, Department of Statistics, Institute of Science, Banaras Hindu University, Varanasi, Uttar Pradesh, India. \\ ${ }^{3}$ Additional Director General (Retired), Central Statistics Office, Ministry of Statistics and Programme Implementation, \\ Government of India, New Delhi, India. \\ DOI: https://doi.org/10.24321/0019.5138.202169
}

I $\quad \begin{array}{lllll}\mathbf{N} & \mathbf{F} & \mathbf{O}\end{array}$

Corresponding Author:

Brijesh P Singh, Department of Statistics, Institute of Science, Banaras Hindu University, Varanasi, Uttar Pradesh, India.

E-mail Id:

brijesh@bhu.ac.in

Orcid Id:

https://orcid.org/0000-0002-2429-4758

How to cite this article:

Chaurasia AR, Singh BP, Singh R. Progression of COVID-19 Pandemic in India: A Linear Functional Concurrent Regression Analysis Approach. J Commun Dis. 2021;53(4):15-22.

Date of Submission: 2021-08-13

Date of Acceptance: 2021-11-25
$\begin{array}{llllllll}\mathbf{A} & \mathbf{B} & \mathbf{S} & \mathbf{T} & \mathbf{R} & \mathbf{A} & \mathbf{C} & \mathbf{T}\end{array}$

Background: COVID-19 is a disasterous pandemic that the world has ever faced. It is affecting the global health system irrespective of race, ethnicity, environment, and economic status. This study is conducted with the aim of assessing the progression of the COVID-19 pandemic in India.

Methods: This article uses the functional concurrent regression analysis approach to describe the pattern of daily reported confirmed cases of COVID-19 in India. The approach provides an excellent fit to the daily reported confirmed cases of the disease. The data used in this study have been taken from covid19india.org.

Results: Estimated value of the parameter $b_{k}$ of the model is highly volatile. During the first phase of the pandemic which last up to 31st March 2020, value was very high. During 31st March to 19th July 2020 except for a few exceptions. Its value again increased rapidly from 17 th February 2021 to 16th April 2021 and started decreasing after midMarch, 2021 and continued decreasing till present.

Conclusion: The data-driven approach used in this study is purely empirical and does not make any assumption about the progression of the pandemic or about the data. The article suggests that based on the parameter of the model, an early warning system may be developed and institutionalised to undertake the necessary measures to control the spread of the disease, thereby controlling the pandemic.

Keywords: CoVID-19, India, Functional Concurrent Regression Analysis, Estimation 


\section{Introduction}

There are many studies related to the modelling of the COVID-19 pandemic in India since the outbreak of the pandemic in the early $2020 .^{1-3}$ These studies have generally followed the epidemiological modelling approach which is primarily based on the basic Susceptible-Infected-Removed (SIR) model and its numerous extensions. ${ }^{4,5}$ The basic SIR model divides the entire population into three mutually exclusive states-Susceptible, Infectious and Removed (by recovery or death). The underlying assumption of the model is that a person once infected and subsequently recovered has little probability to become susceptible to the infection again. ${ }^{6}$ Fitting of the SIR model and its different variants requires defining the transition rate between different states before hand. The progression of the pandemic in the model is characterised in terms of the basic reproduction number denoted by $R_{o}$ (pronounced $R$ nought or $R$ zero) which is the expected number of infected persons directly generated by an infected person in the population where it is assumed that all members of the population are susceptible to the infection. ${ }^{7,8}$ Time-dependent SIR models have also been developed which take into account those infected persons who remain undetected. ${ }^{9}$ The stochastic version of the model has been used to analyse the pace of virus transmissibility by estimating $R_{0}$ using the stochastic Markov Chain Monte Carlo method. ${ }^{10}$

There are also studies that have used different statistical tools and techniques to understand the propagation of the pandemic and suggest afuture course of action. ${ }^{11-14}$ Progression of the pandemic has been analysed using statistical exponential growth model and adopting the serial interval from Severe Acute Respiratory Syndrome. ${ }^{15,16}$ A three-parameter logistic growth function was used to predict the progression of the pandemic for China and for some other countries. ${ }^{17}$ In the context of India, ${ }^{18}$ it is concluded that the nationwide lockdown enforced on March 24, 2020 for 21 days was insufficient for controlling the spread of the pandemic. Mathematical modelling has also been used to describe the progression of the pandemic. ${ }^{19-29}$ Most of these modelling exercises followed an exponential curve during the initial stages of the pandemic which, eventually, was assumed to flattened..$^{30}$ In one study, a stochastic mathematical model is used to describe the progression of COVID-19 pandemic in India. ${ }^{31}$ The logistic growth regression model has also been used to estimate the final size and the peak of the pandemic in many countries and results have been found to be similar to those obtained by the SIR model. ${ }^{32}$ Studies carried out by Sharma et al., Swain et al., Newtonraj et al. ${ }^{33-35}$ revealed that ARIMA model is also appropriate for forecasting COVID-19 in India. Mishra et al. ${ }^{36}$ have advocated SARIMA along with ARIMA for the modelling and forecasting of COVID-19 pandemic whereas Bedi P et al. ${ }^{37}$ have used SEIRD and LSTM models.

In this article, we have followed a data-driven approach to describe the progression of the COVID-19 pandemic in India since March 2020. Data from published epidemiology and virologic studies provide the evidence that COVID-19 is transmitted from an infected person to other persons for a specific period by direct contact with the infected person, or by contact with contaminated objects and surfaces. ${ }^{38}$ Since objects and surfaces are also contaminated by infected persons, the underlying assumption of the present analysis is that the reported number of new cases of COVID-19 is a function of the reported number of new cases of the disease during an immediately preceding period. The World Health Organization has also recommended that in general, a person infected with Novel Coronavirus, the virus that causes COVID-19 disease, may be released from isolation after a period of 13 days based on clinical criteria. ${ }^{39}$ For example, if a person has symptoms for three days, then the person can be released from isolation after 10 days +3 days $=13$ days from the date of onset of symptoms. This, essentially means that there is very little chance that an infected person can infect other persons who come in contact with the infected person after a period of 13 days since infection. It has also been observed that the period between the onset of the symptoms of the disease and the confirmation of the disease through laboratory testing and subsequent reporting is around 3 days. Based on the above evidence, it can be assumed that the progression of the COVID-19 pandemic may be described by establishing a functional relationship between the reported numbers of new confirmed cases of the disease on a particular day with the number of reported numbers of new confirmed cases of the disease during the immediately preceding 10 days period. In this article, we have followed this line of reasoning to describe the progression of the COVID-19 pandemic in India and found that our approach has quite successfully been able to describe the progression of the pandemic in the country.

\section{Data}

The analysis is based on daily reported number of new confirmed cases of COVID-19 disease in India till 11th August, $2021 .^{40}$ The confirmation was done through laboratory testing using the RT-PCR test as recommended by the World Health Organization. An examination of the data released by the Government of India, however, suggests that there are many inconsistencies of known and unknown origin in the daily reporting of new confirmed cases of the disease. The testing for the novel coronavirus in India is voluntary, not population-wide. This means that persons who have not volunteered for testing for novel coronavirus but are positive are not included in the reported number of new confirmed cases of COVID-19 which implies that 
the actual number of new cases of the disease COVID-19 in India may be substantially higher than the reported number of new confirmed cases of the disease released by the Government of India. Moreover, the reported number of new confirmed cases of COVID-19 is also influenced by the number of persons tested for novel coronavirus.

A decrease in the number of persons tested for the virus affects the number of new confirmed cases of the disease reported. In fact, an examination of the time series of the reported number of new confirmed cases released by the Government of India also suggests that there is a dip in the reported number of new confirmed cases of the disease on Monday of almost every week. To minimise the errors associated with daily reporting of new confirmed cases of the disease, it is usually the practice to use 7 days moving average for analysing the progression of the pandemic instead of daily reported new confirmed cases of the disease. The same practice has also been adopted in the present analysis. For example, the reported number of new cases of the disease on 15th August 2020 is actually the simple average of the daily reported new confirmed cases of the disease during the period 12 August 2020 through 18 August 2020.

\section{Method}

We use linear functional concurrent regression modelling approach to analyse the progression of the COVID-19 pandemic in India. Concurrent models are also termed as the varying-coefficient model. ${ }^{41-43}$ These models are particularly useful in the analysis of longitudinal data as is the case here. The linear functional concurrent regression model can be fitted through regression and smoothing spline methods. ${ }^{24,44-45}$ A detailed exposition of linear functional concurrent models and different fitting procedures for these models is given elsewhere. ${ }^{46}$

In what follows, let the entire period of the pandemic is divided into concurrent time segments of 10 days each so that $Y_{j k}$ denotes the reported number of new confirmed cases of the disease on day $j$ of the time segment $k, j$ $=1,2, \ldots 10$. Then the reported number of new confirmed cases of the disease during the time segment $k+1$ may be related to the number of confirmed cases of the disease reported during the time segment $k$ as follows:

$\frac{Y_{j k+1}-\bar{Y}_{j k+1}}{\sigma_{k+1}}=r_{k}\left(\frac{Y_{j k}-\bar{Y}_{k}}{\sigma_{k}}\right)$

Where $r_{k}$ is the correlation coefficient between $Y_{j k}$ and $Y_{j k+1}, j=1,2, \ldots 10, \bar{Y}_{k}$ denotes the average of the reported number of new confirmed cases of the disease during the time segment $k$, and $\sigma_{k}$ is the standard deviation. It may be clarified here that different time segments used for analysing the progression of the pandemic have overlapping days. For example, $k=1$ indicates day 1 through day 10 of the pandemic while $k=2$ indicates day 2 through day 11 .
Equation (1) can be rearranged as,

$Y_{j k+1}=\left(\bar{Y}_{k+1}-b_{k} \bar{Y}_{k}\right)+b_{k} Y_{j k}$

Where $b_{k}=r \frac{\sigma_{k+1}}{\sigma_{k}}$

Equation (3) suggests that when $Y_{j k+1}=b_{k} Y_{j k}$ for all $j$ $=1,2, \ldots 10, \bar{Y}_{k+1}-b_{k} \bar{Y}_{k}=0$.In this ideal situation, the reported number of new confirmed cases of the disease during the time segment $j+1$ is $b_{k}$ times the reported number of new confirmed cases of the disease during the time segment $k$. This essentially means that all reported new confirmed cases of the disease in the time segment $k+1$ are known contacts of the reported number of new confirmed cases of the disease in the time segment $k$. It is, however, rare that $\bar{Y}_{k+1}-b_{k} \bar{Y}_{k}=0$ for two reasons. First, all known contacts of the reported new confirmed cases of disease during the time segment $k$ are not reported during the time segment $k+1$. Second, there may be confirmed cases of unknown contacts who are reported during the time segment $k+1$. When $\bar{Y}_{k+1}-b_{k} \bar{Y}_{k}>0$, there are some confirmed cases of unknown contacts of the disease reported in the time segment $k+1$. On the other hand, when $\bar{Y}_{k+1}-b_{k} \bar{Y}_{k}<0$ , there are some known contacts of the confirmed cases of the disease reported during the time segment $k$ who are not reported during the time segment $k+1$.

Model (2) suggests that the progression of the pandemic can be characterised in terms of the parameter $b_{k}$. When $b_{k}>1, Y_{j k+1}>Y_{j k}, j=1,2, \ldots 10$, which means an increase in the reported number of new confirmed cases of the disease during the time segment $k+1$ relative to the reported number of new confirmed cases of the disease during the time segment $k$. On the other hand, when $b_{k}<1, Y_{j k+1}<Y_{j k}$, $j=1,2, \ldots 10$, there is a decrease in the reported number of new confirmed cases of the disease during the time segment $k+1$ relative to the reported number of new confirmed cases of the disease during the time segment $k$. If all contacts of the reported number of new confirmed cases of the disease reported during the time segment $k$ are traced, tested and positive cases of novel coronavirus are identified and reported, then the reported number of new confirmed cases of the disease during the time segment $k+1$ can be determined from the reported number of new confirmed cases of the disease during the time segment $k$. Model (2) serves as a data-driven model for analysing the progression of COVID-19 pandemic. The model does not make any assumptions about the progression of the disease. It is based on the relationship between the numbers of new confirmed cases of the disease reported during two concurrent time segments of 10 days.

\section{Results}

Figure 1 presents the trend in the estimated value of the parameter $b_{k}$ of model (2) from 11th March 2020 to 11th August 2021. The trend is highly volatile. The parameter 
$b_{k}$ is the key factor in deciding the pace of progression of the pandemic. During its early days, COVID-19 pandemic in India progressed very rapidly. The first phase of the pandemic appears to have lasted till 31st March 2020. During this phase, the number of new confirmed cases of the disease increased from an estimated 7 cases on 11th March 2020 to estimated 426 cases on 31st March 2020 or the reported new confirmed cases of the disease multiplied more than 60 times during this period.

The second phase of the pandemic appears to have started around 31st March 2020 and lasted till 19th July 2020. During this period, the estimated value of $b_{k}$ has always been more than 1 but less than 2 except for a few exceptions when it was less than 1 and for a few exceptions when it was more than 2 but always less than 3 . This means that except for a few days during this period, there has been an increase in the reported number of new confirmed cases of the disease but the pace of the increase in the reported number of new confirmed cases of the disease was not fast as the estimated $b_{k}$ was rarely more than 2 during this period.

The third phase of the pandemic appears to have started around 20th July 2020 and lasted up to 15th August 2020. During this period, barring a few exceptions, the estimated $b_{k}$ was always less than 1 indicating a decrease in the daily reported number of new confirmed cases of the disease. This period was, however, followed by a period of spike in $b_{k}$ till 31st August 2020. During this period, the estimated $b_{k}$ was always more than 1 and even increased to more than 4 in some days leading to a rapid increase in the reported number of new confirmed cases of the disease. This period was followed by a rapid decrease in the estimated value of $b_{k}$ up to 18th September 2020. The rapid decrease in estimated $b_{k}$ during this period resulted in a rapid decrease in the daily reported number of new confirmed cases of the disease. However, $b_{k}$ increased again from 26th September 2020 to 16 th October 2020, but the increase in the daily reported number of new cases of the disease was not very rapid as estimated $b_{k}$ was never more than 2 during this period.

The next phase of the pandemic appears to have started from 17th October 2020 and lasted up to 15th February 2021. This period is characterised by a decrease in the number of daily confirmed cases of the disease as estimated $b_{k}$ remained less than 1 in most of the days of this period so that there was a digression of the pandemic. However, $b_{k}$ increased rapidly from 17th February 2021 to16th April 2021 so that there was a very rapid increase in the daily reported number of new confirmed cases of the disease. Although $b_{k}$ started decreasing after mid-March, 2021 yet, $b_{k}$ decreased to less than 1 only in mid-April 2021 resulting in a decrease in the daily reported number of new confirmed cases of the disease during the time segment $k+1$ relative to the time segment $k$.

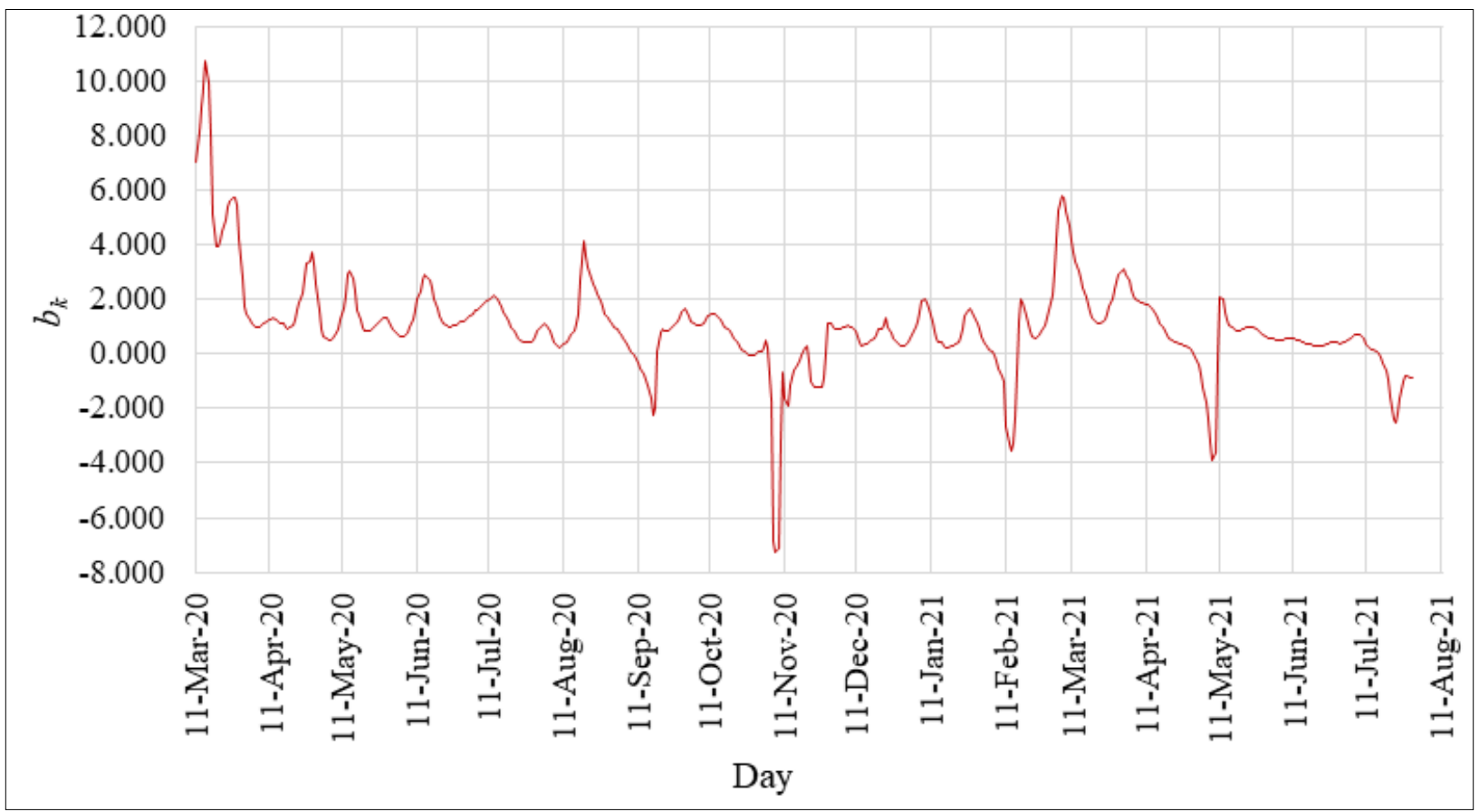

Figure I.Trend in Estimated Values of $\boldsymbol{b}_{\boldsymbol{k}}$

Source: Estimated by authors from the daily reported number of new confirmed cases of the disease in conjunction with model (2). 


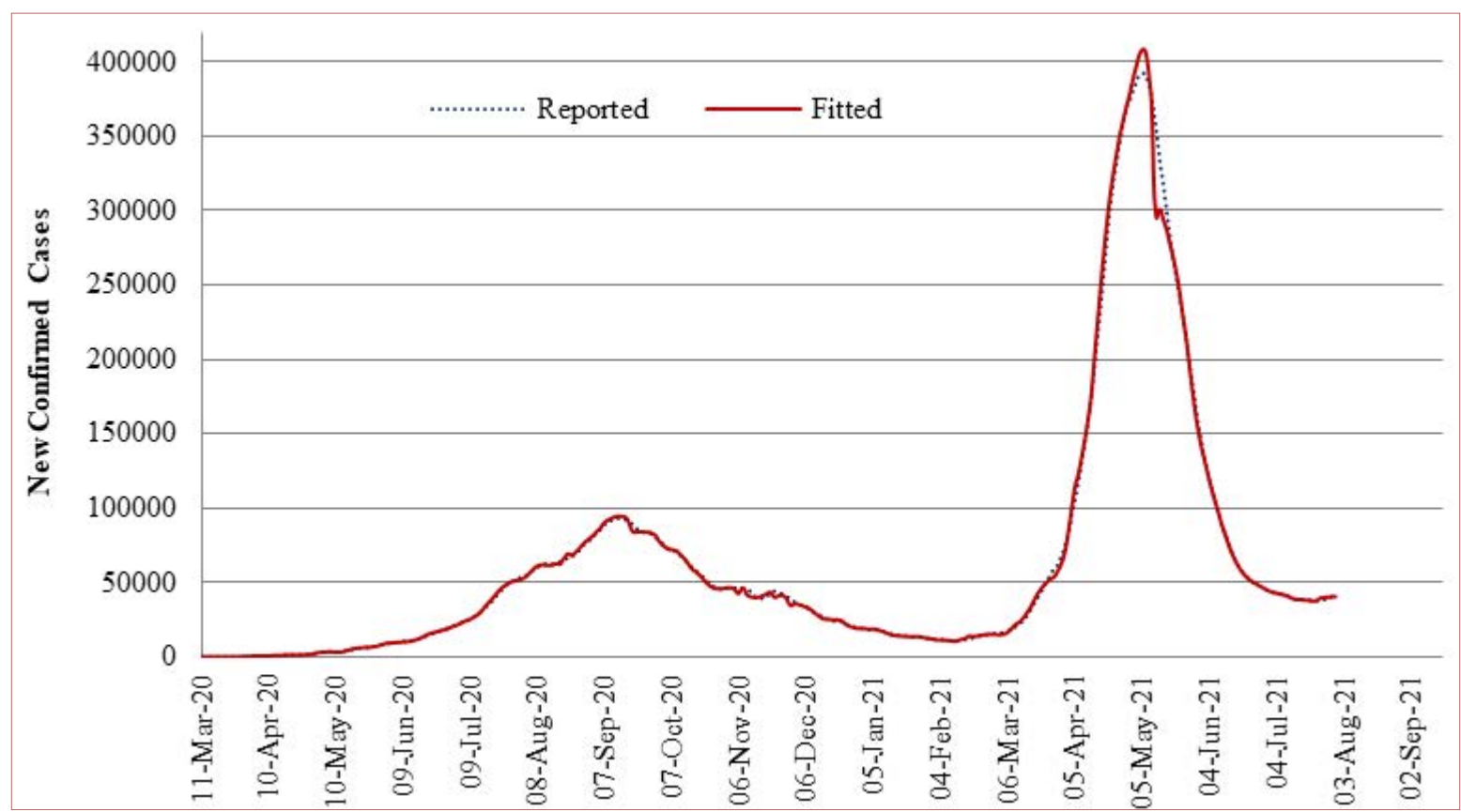

Figure 2.Reported and Fitted Reported Number of New Confirmed Cases ofCOVID-I 9 in India

Source: Reported number of new confirmed cases of the disease is released by the Government of India and are available at https://www.covid19india.org. Fitted number of new confirmed cases of the disease is calculated by authors in conjunction with model (2).

The daily reported number of new confirmed cases of the disease is also influenced by the difference $\bar{Y}_{k+1}-b_{k} \bar{Y}_{k}$. If this difference is greater than 0 , then the daily reported number of new confirmed cases of the disease is more than that implied by the estimated $b_{k}$. For example, estimated $b_{k}=1.020$ between the time segment beginning 16 th June 2020 and the immediately succeeding time segment beginning 26th June 2020 . This means that the number of new confirmed cases of the disease reported on 26th June 2020 should be 1.020 times the number of new confirmed cases of the disease reported on 16th June 2020 (12320). However, the number of new confirmed cases of the disease, actually reported on 26th June 2020, was only 18154. This implies that an estimated 5572 new confirmed cases of the disease reported on 26th June 2020 were the cases of unknown contacts, although a part of this difference may also be due to reporting and other inconsistencies. On the other hand, if the difference $\bar{Y}_{k+1}-b_{k} \bar{Y}_{k}$ is less than 0 , then this implies that all new confirmed cases of the disease implied by $b_{k}$ are not reported. This means that all contacts of the new confirmed cases of the disease reported during the time segment $k$ could not be traced/tested and positive cases reported during the time segment $k+1$.

Figure 2 depicts the daily reported number of new confirmed cases of COVID-19 in India beginning 1st March 2020 through 11th August 2021 along with the fitted values of the daily reported number of new confirmed cases of the disease which have been obtained from the estimated $b_{k}$ in conjunction with model (2). It is evident from the figure that model (2) fits very well with the daily reported number of new confirmed cases of the disease. More specifically, the model effectively captures the very rapid increase in the daily reported number of new confirmed cases of the disease in the recent past reflecting the second wave of the COVID-19 pandemic in the country.

\section{Conclusion}

This article has followed a data-driven approach to analyse and describe the progression of the COVID-19 pandemic in India. The approach is purely empirical and does not make any assumptions about the progression of the pandemic or about the data. Figure 2 empirically validates the datadriven approach adopted in this article. The present analysis suggests that two factors may be attributed to the progression of the COVID-19 pandemic in India. The first, obviously, is the multiplicity of the reported new confirmed cases of the disease which has been captured through the parameter $b_{k}$ of the model (2). The second factor in the progression of the pandemic in the country appears to be the inability to contact and trace all contacts of daily reported new confirmed cases of the disease and their testing to identify the cases of the disease. This inability has resulted in a large number of reported new confirmed cases of the disease with unknown contacts.

Our analysis suggests that keeping a close watch on the trend in the parameter $b_{k}$ may give an early indication about the increase in the number of new confirmed cases of the disease. An exceptional increase in $b_{k}$ is an indication of 
some exogenous factors that may be responsible for the increase in the multiplicity of the reported number of new confirmed cases of the disease such as a new mutation or strain of the novel coronavirus. It is possible to forecast the daily reported number of new confirmed cases of the disease by projecting $b_{k}$ using appropriate forecasting tools such as trend analysis or time series analysis. It may, however, be noted that the trend in the parameter $b_{k}$ has been found to be highly volatile so that the long-term projection of the parameter $b_{k}$ does not appear to be a feasible proposition. Because of the high volatility of the parameter $b_{k}$, conventional forecasting tools such as linear regression analysis may not be appropriate. Instead, techniques such as joinpoint regression may be used for this purpose. ${ }^{21}$ In any case, very high volatility of the parameter $b_{k}$ probably and so obviously appears to be the reason why most of the existing approaches have not been successful in forecasting the progression of the pandemic.

\section{Contribution}

ARC conceptualised the study, developed the model and carried out calculations.

BPS carried out literature and model review and read the manuscript.

RS read the manuscript.

\section{Source of Funding}

The present research did not receive any grant from funding sources such as public, commercial or NGOs.

\section{Conflict of Interest: None}

\section{References}

1. Kumar P, Erturk VS. A case study of Covid-19 epidemic in India via generalised Caputo type fractional derivatives. Math Methods Appl Sci. 2021 Feb;10.1002/mma.7284. [PubMed] [Google Scholar]

2. Banerjee R, Bhattacharjee S, Varadwaj PK. Analyses and forecasts for COVID-19 in India. MedRxiv.2020. [Google Scholar]

3. Biswas SK, Ghosh JK, Sarkar S, Ghosh U. COVID-19 pandemic in India: a mathematical model study. NonlinearDyn.2020 Sep;102:537-53. [PubMed] [Google Scholar]

4. Smith D, Moore L [Internet]. The SIR model for spread of disease: The differential equation model. Convergence; 2004. Available from: https://www.maa.org/press/ periodicals/loci/joma/the-sir-model-for-spread-ofdisease-the-differential-equation-model. [Google Scholar]

5. Allen LJ, Brauer F, Van den Driessche P, Wu J. Mathematical epidemiology. Vol. 1945. Springer;2008. [Google Scholar]

6. Kermack WO, McKendrick AG. A contribution to the mathematical theory of epidemics. Proc Roy Soc Lond. 1927;115:700-21. [Google Scholar]

7. Fraser C, Donnelly CA, Cauchemez S, Hanage WP, Van Kerkhove MD, Hollingsworth TD. Pandemic potential of a strain of influenza $A(H 1 N 1)$ : early findings. Science. 2009;324(5934):1557-61. [Google Scholar]

8. Milligan GN, Barrett AD. Vaccinology: an essential guide. John Wiley \& Sons;2014. [Google Scholar]

9. Chen YC, Lu PE, Chang CS, Liu TH. A time-dependent SIR model for COVID-19 with undetectable infected persons. IEEE Trans Net Sci Eng. 2020;7(4):3279-94. [Google Scholar]

10. Wu JT, Leung K, Leung GM. Now casting and forecasting the potential domestic and international spread of the 2019-nCoV outbreak originating in Wuhan, China: a modelling study. Lancet. 2020 Feb;395(10225):689-97. [PubMed] [Google Scholar]

11. Batista M. Estimation of the final size of the second phase of the Corona virus COVID 19 epidemic by the logistic model. MedRxiv.2020. [Google Scholar]

12. Koo JR, Cook AR, Park M, Sun Y, Sun H, Lim JT, Tam C, Dickens BL.Interventions to mitigate early spread of SARS-CoV-2 in Singapore: a modelling study. Lancet Infect Dis. 2020 Jun;20:678-88. [PubMed] [Google Scholar]

13. Kucharski AJ, Russell TW, Diamond C, Liu Y, Edmunds J, Funk S, Eggo RM; Centre for Mathematical Modelling of Infectious Diseases COVID-19 working group.Early dynamics of transmission and control of COVID-19: a mathematical modelling study. Lancet Infect Dis. 2020;20(5):553-8. [PubMed] [Google Scholar]

14. Tuite AR, Fisman DN. Reporting, epidemic growth, and reproduction numbers for the 2019 novel Corona virus (2019-nCoV) epidemic. Ann Intern Med. 2020 Apr;172(8):567-68. [PubMed] [Google Scholar]

15. Zhao S, Lin Q, Ran J, Musa SS, Yang G, Wang W, Lou Y, Gao D, Yang L, He D, Wang MH.Preliminary estimation of the basic reproduction number of novel Corona virus (2019-nCoV) in China, from 2019 to 2020: a data-driven analysis in the early phase of the outbreak. Int J Infect Dis. 2020 Mar;92:214-7. [PubMed] [Google Scholar]

16. Roy TK, Singh BP. A data driven model for prediction of COVID-19 outbreak in Bangladesh. Bangladesh J Infect Dis.2020;7(1):22-6. [Google Scholar]

17. Shen CY. A logistic growth model for COVID-19 proliferation: experiences from China and international implications in infectious diseases. IntJ Infect Dis.2020;96:582-9.

18. Singh R, Adhikari R. Age-structured impact of social distancing on the COVID-19 epidemic in India. arXiv. 2020;12055. [Google Scholar]

19. Rao Srinivasa ASR, Krantz Steven G, Thomas K, Bhat R. Model-based retrospective estimates for COVID-19 
or Corona virus in India: continued efforts required to contain the virus spread. Curr Sci. 2020;118(7):102325. [Google Scholar]

20. Chang SL, Harding N, Zachreson C, Cliff OM, Prokopenko M. Modelling transmission and control of the COVID-19 pandemic in Australia. Nat Commun.2020 Nov;11(1):113. [PubMed] [Google Scholar]

21. Chaurasia AR, Singh BP. COVID-19 Trend and forecast in India: ajoin point regression analysis. Demog India. 2020;49(Special Issue):15-26. [Google Scholar]

22. Singh BP. Modelling and forecasting the spread of COVID-19 pandemic in India and significance of lockdown: A mathematical outlook. Handbook of Statistics. 2021:257-89. [Google Scholar]

23. Singh BP, Singh G. Modelling tempo of COVID-19 pandemic in India and significance of lockdown. J Public Aff. 2020 Aug;20(4):e2257.[PubMed] [Google Scholar]

24. Huang $C$, Wang $Y$, Li X, Ren $L$, Zhao J, Hu Y, Zhang L, Fan G, Xu J, Gu X, Cheng Z, Yu T, Xia J, Wei Y, Wu W, Xie X, Yin W, Li H, Liu M, Xiao Y, Gao H, Guo L, Xie J, Wang G, Jiang R, Gao Z, Jin Q, Wang J, Cao B.Clinical features of patients infected with 2019 novel corona virus in Wuhan, China. Lancet. 2020 Feb;395(10223):497-506. [PubMed] [Google Scholar]

25. Hui DS, I Azhar E, Madani TA, Ntoumi F, Kock R, Dar O, Ippolito G, Mchugh TD, Memish ZA, Drosten C, Zumla A, Petersen E. The continuing 2019-ncov epidemic threat of novel Corona viruses to global health-the latest 2019 novel corona virus outbreak in Wuhan, China. IntJ Infect Dis. 2020 Feb;91:264. [PubMed] [Google Scholar]

26. Corman VM, Landt O, Kaiser M, Molenkamp R, Meijer A, Chu DK, Bleicker T, Brünink S, Schneider J, Schmidt $\mathrm{ML}$, Mulders DG, Haagmans BL, van der Veer $B$, van den Brink S, Wijsman L, Goderski G, Romette JL, Ellis J, Zambon M, Peiris M, Goossens H, Reusken C, Koopmans MP, Drosten C.Detection of 2019 novel Corona virus (2019-ncov) by real time RT-PCR. Eurosurveillance. 2020 Jan;25(3):2000045. [PubMed] [Google Scholar]

27. Rothe C, Schunk M, Sothmann P, Bretzel G, Froeschl G, Wallrauch C, Zimmer T, Thiel V, Janke C, Guggemos W, Seilmaier M, Drosten C, Vollmar P, Zwirglmaier K, Zange S, Wölfel R, Hoelscher M.Transmission of 2019-ncov infection from an asymptomatic contact in Germany. N Engl J Med. 2020;382(10):970-1. [PubMed] [Google Scholar]

28. Anastassopoulou C, Russo L, Tsakris A, Siettos C. Data-based analysis, modelling and forecasting of the COVID-19 outbreak. PLoS ONE.2020 Mar;15(3):e0230405. [PubMed] [Google Scholar]

29. Gamero J, Tamayo JA,Martinez-Roman JA. Forecast of the evolution of the contagious disease caused by novel corona virus (2019-ncov) in China.
arXiv.2020;2002:04739. [Google Scholar]

30. Junling M, Dushoff J, Bolker BM, Earn DJ. Estimating initial epidemic growth rates. Bull Math Biol. 2014 Jan;76(1):245-60. [PubMed] [Google Scholar]

31. Chatterjee K, ChatterjeeK, Kumar A, Shankar S. Healthcare impact of COVID-19 epidemic in India: astochastic mathematical model. Med J Armed Forces India. 2020 Apr;76(2):147-55. [PubMed] [Google Scholar]

32. Batista M. Estimation of the final size of the coronavirus epidemic by the SIR model. University of Ljubljana, Slovenia;2020. [Google Scholar]

33. Sharma VK, Nigam U. Modelling and forecasting of COVID-19 growth curve in India. Trans Indian Natl Acad Eng. 2020;5:697-710. [Google Scholar]

34. Swain PK, Tripathy MR, Jena D, Fenta HM, Zike DT. Modelling and forecasting of COVID-19 cases in Odisha and India. Demog India. 2020;49(Special Issue):66-75. [Google Scholar]

35. Newtonraj A, Mani M. Autoregressive integrated moving average model for forecasting COVID-19 in India. J Postgrad Med. 2020;54(3):122-5. [Google Scholar]

36. Mishra P, Al Khatib AMG, Sardar I, Mohammed J, Ray M, Kumar M, Rawat D, Pandey SA, Dubey A, Feys J, Rono K. Modelling and forecasting of COVID-19 in India. J InfectDis Epi. 2020;6:162. [Google Scholar]

37. Bedi P, Dhiman S, Gole P, Gupta N, Jindal V. Prediction of COVID-19 trend in India and its four worst-affected states using modified SEIRD and LSTM models. SN Comput Sci.2021;2:224. [PubMed] [Google Scholar]

38. World Health Organization[Internet]. Report of the WHO-China Joint Mission on Corona virus Disease 2019 (COVID-19) 16-24 February 2020. Geneva: World Health Organization; 2020. Available from: https:// www.who.int/docs/default-source/Corona viruse/ who-china-joint-mission-on-covid-19-finalreport.pdf

39. World Health Organization [Internet]. Criteria for releasing COVID-19 patients from isolation. Geneva: World Health Organization; 2020. Available from: https://www.who.int/news-room/commentaries/ detail/criteria-for-releasing-covid-19-patients-fromisolation.

40. COVID-19 India [Internet]; 2021.Available from: https:// www.covid19india.org

41. Fan J, Zhang W. Statistical estimation in varying coefficient models. Ann Statist. 1999;27:1491-518. [Google Scholar]

42. Fan J, Zhang W. Statistical methods with varying coefficient models. Stat Interface. 2008;1(1):179-95. [PubMed] [Google Scholar]

43. Şentürk D, Müller HG. Functional varying coefficient models for longitudinal data. J Am Stat 
Assoc.2010;105(491):1256-64. [Google Scholar]

44. Eubank RL, Huang C, Mũnoz Maldonado Y, Wang N, Wang S, Buchanan RJ. Smoothing spline estimation in varying-coefficient models. JR Stat Soc Series B Stat Methodol.2004;66:653-67. [Google Scholar]

45. Huang JZ, Wu CO, Zhou L. Varying-coefficient models and basis function approximations for the analysis of repeated measurements. Biometrika. 2002;89:111-28. [Google Scholar]

46. Ramsay JO, Silverman BW. Applied functional data analysis: methods and case studies. New York: Springer;2007. [Google Scholar] 